\title{
A unified method for the recovery of metals
}

\section{from chalcogenides}

Francesca Bevan, ${ }^{\text {a }}$ Hanaa Galeb,,${ }^{\text {a,b }}$ Alexander Black, ${ }^{a}$ Ioanna Maria Pateli, a,c Jack Allen, ${ }^{\mathrm{a}}$ Magali Perez, ${ }^{d}$ Jörg Feldmann, ${ }^{d, e}$ Robert Harris, ${ }^{\mathrm{a}}$ Gawen Jenkin, ${ }^{\mathrm{f}}$ Andrew Abbott, ${ }^{\mathrm{a}}$ Jennifer Hartley a*

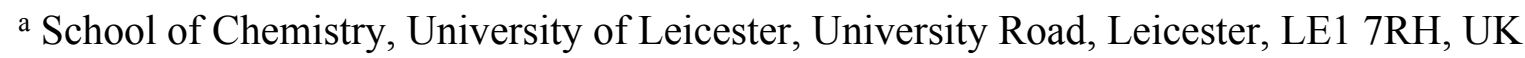

${ }^{\mathrm{b}}$ Department of Chemistry, King Abdulaziz University, 21577 Jeddah, Saudi Arabia

c Stephenson Institute for Renewable Energy, University of Liverpool, Peach Street, Liverpool, L69 7ZF, UK

${ }^{d}$ Department of Chemistry, University of Aberdeen, Meston Walk, Aberdeen, AB24 3UE, UK

${ }^{\mathrm{e}}$ Institute of Chemistry, University of Graz, Universitätsplatz 1/I, 8010 Graz, Austria

${ }^{f}$ School of Geography, Geology and the Environment, University of Leicester, University Road, Leicester, LE1 7RH, UK

Number of pages: 3

Number of figures: 1

Number of tables: 1 


\section{Supporting information}
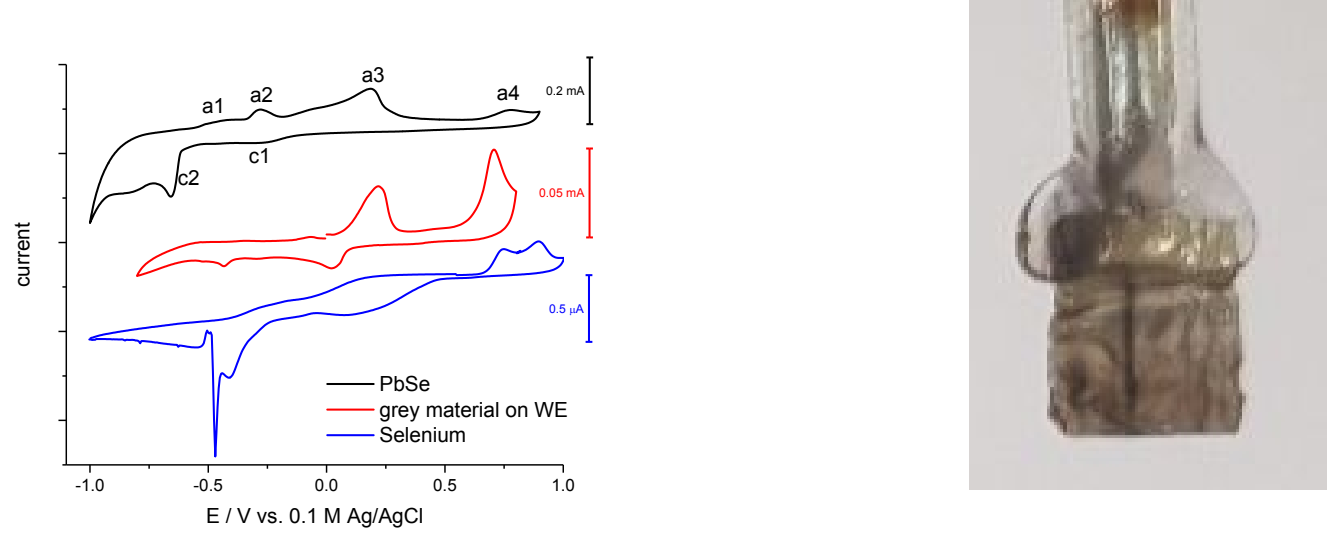

Figure S1. Voltammogram of grey material present on the working electrode after repeated cycling of $\mathrm{PbSe}$, compared to the voltammograms of paint casted $\mathrm{PbSe}$ and elemental selenium (left), and an image of this grey coating (right). 


\begin{tabular}{|c|c|c|}
\hline Chemical & Purity / \% & Source \\
\hline Bismuth chloride $\left(\mathrm{BiCl}_{3}\right)$ & $<98$ & Aldrich \\
\hline Bismuth selenide $\left(\mathrm{Bi}_{2} \mathrm{Se}_{3}\right)$ & $\geq 99.995$ & Sigma Aldrich \\
\hline Bismuth telluride $\left(\mathrm{Bi}_{2} \mathrm{Te}_{3}\right)$ & 99.99 & Sigma Aldrich \\
\hline Copper(I) chloride $(\mathrm{CuCl})$ & $\geq 99$ & Sigma Aldrich \\
\hline Copper(II) chloride $\left(\mathrm{CuCl}_{2}\right)$ & 99 & Acros Organics \\
\hline Copper(I) oxide $\left(\mathrm{Cu}_{2} \mathrm{O}\right)$ & 99.9 & Alfa Aesar \\
\hline Copper(I) sulfide $\left(\mathrm{Cu}_{2} \mathrm{~S}\right)$ & 99.5 & Alfa Aesar \\
\hline Copper(I) selenide $\left(\mathrm{Cu}_{2} \mathrm{Se}\right)$ & 99.95 & Sigma Aldrich \\
\hline Copper(I) telluride $\left(\mathrm{Cu}_{2} \mathrm{Te}\right)$ & 99.5 & Alfa Aesar \\
\hline Lead(II) chloride $\left(\mathrm{PbCl}_{2}\right)$ & 98 & Aldrich \\
\hline Lead(II) oxide $(\mathrm{PbO})$ & 99.999 & Alfa Aesar \\
\hline Lead(II) sulfide (PbS) & 99.9 & Aldrich \\
\hline Lead(II) selenide (PbSe) & $99.999+$ & Alfa Aesar \\
\hline Lead(II) telluride (PbTe) & 99.99 & Alfa Aesar \\
\hline Silver chloride $(\mathrm{AgCl})$ & 99.9 & Alfa Aesar \\
\hline Silver sulfide $\left(\mathrm{Ag}_{2} \mathrm{~S}\right)$ & n.a. & Chem Cruz \\
\hline Silver selenide $\left(\mathrm{Ag}_{2} \mathrm{Se}\right)$ & n.a. & Aldrich \\
\hline Silver telluride $\left(\mathrm{Ag}_{2} \mathrm{Te}\right)$ & n.a. & Aldrich \\
\hline Sodium sulfide nonahydrate $\left(\mathrm{Na}_{2} \mathrm{~S}\right)$ & $\geq 98$ & Honeywell \\
\hline Sodium selenide $\left(\mathrm{Na}_{2} \mathrm{Se}\right)$ & 99.8 & Alfa Aesar \\
\hline Sodium selenite $\left(\mathrm{Na}_{2} \mathrm{SeO}_{3}\right)$ & 99 & Sigma Aldrich \\
\hline Sodium selenate $\left(\mathrm{Na}_{2} \mathrm{SeO}_{4}\right)$ & $99.8+$ & Alfa Aesar \\
\hline Sodium telluride $\left(\mathrm{Na}_{2} \mathrm{Te}\right)$ & 99.9 & Alfa Aesar \\
\hline Sodium tellurite $\left(\mathrm{Na}_{2} \mathrm{TeO}_{3}\right)$ & 99 & Alfa Aesar \\
\hline Sodium tellurate $\left(\mathrm{Na}_{2} \mathrm{TeO}_{4}\right)$ & 99 & Sigma Aldrich \\
\hline Zinc chloride $\left(\mathrm{ZnCl}_{2}\right)$ & $>98$ & Sigma Aldrich \\
\hline Zinc sulfide $(\mathrm{ZnS})$ & 99.99 & Aldrich \\
\hline Zinc selenide $(\mathrm{ZnSe})$ & 99.99 & Alfa Aesar \\
\hline Zinc telluride (ZnTe) & 99.99 & Alfa Aesar \\
\hline
\end{tabular}

